\title{
ACCORD: A Multicentre, Seamless, Phase 2 Adaptive Randomisation Platform Study to Assess the Efficacy and Safety of Multiple Candidate Agents for the Treatment of COVID-19 in Hospitalised Patients: A structured summary of a study protocol
for a randomised controlled trial
}

Tom Wilkinson ${ }^{1,2}$, Rupert Dixon ${ }^{3}$, Clive Page ${ }^{4}$, Miles Carroll ${ }^{5}$, Gareth Griffiths ${ }^{1 *}$, Ling-Pei Ho ${ }^{6}$, Anthony De Soyza ${ }^{7}$, Timothy Felton ${ }^{8}$, Keir E. Lewis ${ }^{9}$, Karen Phekoo ${ }^{10}$, James D. Chalmers ${ }^{11}$, Anthony Gordon ${ }^{12}$, Lorcan McGarvey ${ }^{13}$, Jillian Doherty ${ }^{10}$, Robert C. Read ${ }^{1,2}$, Manu Shankar-Hari ${ }^{4}$, Nuria Martinez-Alier ${ }^{3,14}$, Michael O'Kelly ${ }^{15}$, Graeme Duncan ${ }^{16}$, Roelize Walles ${ }^{3}$, James Sykes ${ }^{3}$, Charlotte Summers ${ }^{17}$, Dave Singh ${ }^{18}$ and on behalf of the ACCORD Collaborators

\begin{abstract}
Objectives: Stage 1: To evaluate the safety and efficacy of candidate agents as add-on therapies to standard of care (SoC) in patients hospitalised with COVID-19 in a screening stage.

Stage 2: To confirm the efficacy of candidate agents selected on the basis of evidence from Stage 1 in patients hospitalised with COVID-19 in an expansion stage.

Trial design: ACCORD is a seamless, Phase 2, adaptive, randomised controlled platform study, designed to rapidly test candidate agents in the treatment of COVID-19. Designed as a master protocol with each candidate agent being included via its own sub-protocol, initially randomising equally between each candidate and a single contemporaneous SoC arm (which can adapt into 2:1). Candidate agents currently include bemcentinib, MEDI3506, acalabrutinib, zilucoplan and nebulised heparin. For each candidate a total of 60 patients will be recruited in Stage 1. If Stage 1 provides evidence of efficacy and acceptable safety the candidate will enter Stage 2 where a total of approximately 126 patients will be recruited into each study arm sub-protocol. Enrollees and outcomes will not be shared across the Stages; the endpoint, analysis and sample size for Stage 2 may be adjusted based on evidence from Stage 1. Additional arms may be added as new potential candidate agents are identified via candidate agent specific sub-protocols.
\end{abstract}

* Correspondence: G.O.Griffiths@soton.ac.uk

${ }^{1}$ University of Southampton, Southampton, Hampshire, UK

Full list of author information is available at the end of the article

(c) The Author(s). 2020 Open Access This article is licensed under a Creative Commons Attribution 4.0 International License, which permits use, sharing, adaptation, distribution and reproduction in any medium or format, as long as you give appropriate credit to the original author(s) and the source, provide a link to the Creative Commons licence, and indicate if changes were made. The images or other third party material in this article are included in the article's Creative Commons licence, unless indicated otherwise in a credit line to the material. If material is not included in the article's Creative Commons licence and your intended use is not permitted by statutory regulation or exceeds the permitted use, you will need to obtain permission directly from the copyright holder. To view a copy of this licence, visit http://creativecommons.org/licenses/by/4.0/. The Creative Commons Public Domain Dedication waiver (http://creativecommons.org/publicdomain/zero/1.0/) applies to the data made available in this article, unless otherwise stated in a credit line to the data. 
(Continued from previous page)

Participants: The study will include hospitalised adult patients ( $\geq 18$ years) with confirmed SARS-CoV-2 infection, the virus that causes COVID-19, that clinically meet Grades 3 (hospitalised - mild disease, no oxygen therapy), Grades 4 (hospitalised, oxygen by mask or nasal prongs) and 5 (hospitalised, non-invasive ventilation or high flow oxygen) of the WHO Working Group on the Clinical Characteristics of COVID-19 9-point category ordinal scale. Participants will be recruited from England, Northern Ireland, Wales and Scotland.

Intervention and comparator: Comparator is current standard of care (SoC) for the treatment of COVID-19. Current candidate experimental arms include bemcentinib, MEDI3506, acalabrutinib, zilucoplan and nebulised heparin with others to be added over time. Bemcentinib could potentially reduce viral infection and blocks SARS-CoV-2 spike protein; MEDI3506 is a clinic-ready anti-IL-33 monoclonal antibody with the potential to treat respiratory failure caused by COVID; acalabrutinib is a BTK inhibitor which is anti-viral and anti-inflammatory; zilucoplan is a complement C5 inhibitor which may block the severe inflammatory response in COVID-19 and; nebulised heparin has been shown to bind with the spike protein. ACCORD is linked with the UK national COVID therapeutics task force to help prioritise candidate agents.

Main outcomes: Time to sustained clinical improvement of at least 2 points (from randomisation) on the WHO 9point category ordinal scale, live discharge from the hospital, or considered fit for discharge (a score of 0, 1, or 2 on the ordinal scale), whichever comes first, by Day 29 (this will also define the "responder" for the response rate analyses).

Randomisation: An electronic randomization will be performed by Cenduit using Interactive Response Technology (IRT). Randomisation will be stratified by baseline severity grade. Randomisation will proceed with an equal allocation to each arm and a contemporaneous SoC arm (e.g. 1:1 if control and 1 experimental arm; 1:1:1 if two experimental candidate arms etc) but will be reviewed as the trial progresses and may be changed to 2:1 in favour of the candidate agents.

Blinding (masking): The trial is open label and no blinding is currently planned in the study.

Numbers to be randomised (sample size): This will be in the order of 60 patients per candidate agent for Stage 1, and 126 patients for Stage 2. However, sample size re-estimation may be considered after Stage 1. It is estimated that up to 1800 patients will participate in the overall study.

Trial Status: Master protocol version ACCORD-2-001 - Master Protocol (Amendment 1) $22^{\text {nd }}$ April 2020, the trial has full regulatory approval and recruitment is ongoing in the bemcentinib (first patient recruited 6/5/2020), MEDI3506 (first patient recruited 19/5/2020), acalabrutinib (first patient recruited 20/5/2020) and zilucoplan (first patient recruited 19/5/2020) candidates (and SoC). The recruitment dates of each arm will vary between candidate agents as they are added or dropped from the trial, but will have recruited and reported within a year.

Trial registration: EudraCT 2020-001736-95, registered 28 ${ }^{\text {th }}$ April 2020.

Full protocol: The full protocol (Master Protocol with each of the candidate sub-protocols) is attached as an additional file, accessible from the Trials website (Additional file 1). In the interest in expediting dissemination of this material, the familiar formatting has been eliminated; this Letter serves as a summary of the key elements of the full protocol.

Keywords: COVID-19, randomised, platform study, master protocol, phase II 


\section{Supplementary information}

Supplementary information accompanies this paper at https://doi.org/10. 1186/s13063-020-04584-9.

\section{Additional file 1.}

\section{Acknowledgements}

We acknowledge the University Hospital Southampton NHS Foundation Trust who are the sponsor for ACCORD. We also acknowledge the involvement of the NIHR community in developing and conducting the ACCORD, including but not limited to the NIHR Respiratory Translational Research Collaboration, NIHR Biomedical Research Centres and NIHR Clinical Research Facilities.

\section{Authors' contributions}

TW, DS, CP, MC, NM-A, MO and RD came up with the concept of the development of ACCORD and oversaw the development of the master protocol and this submission. TW, DS, CP, L-PH, AdS, TF, KL, KP, IC, AG, LM, JD, RR, M$\mathrm{SH}, \mathrm{CS} \& \mathrm{GG}$ are ACCORD steering committee members overseeing the coordination of ACCORD and the protocol. $\mathrm{MO}$ and JS were responsible for the development of the statistical design and with GG the statistical aspects of running and analysing the trial. RW and GD were responsible for developing the trial management and delivery aspects of the trial. All were involved in the development of the master protocol and this submission. The author(s) read and approved the final manuscript.

\section{Funding}

The ACCORD trial platform is funded by UKRI. The funder will have no role in the study's design, collection, management, analysis and interpretation of data, writing of the report and the decision to submit the report for publication conception or in the data analysis.

\section{Availability of data and materials}

When the database from each Candidate sub-protocol is locked, analysed and published we will make the data available to the academic community via www.clinicalstudydatarequest.com.

\section{Ethics approval and consent to participate}

Health Research Authority and Research Ethics Committee, Bristol HRA Centre (REC reference: 20/SC/0201), 24 $4^{\text {th }}$ April 2020

I certify that this trial has received appropriate ethical approval as described above.

We will obtain consent from all participants entering into the ACCORD study.

\section{Consent for publication}

Not applicable

\section{Competing interests}

GD, RR, KP, MC, RD, MS-H, RW, NM-A, JD and JS declare no competing interests. MOK has carried out consulting work for AZ. AdS has received research grants/personal fees/travel from AstraZeneca, Bayer, Boehringer Ingelheim, GlaxoSmithKline, Novartis, Pfizer and Medimmune. AG has received consulting fees paid to institution from GlaxoSmithKline, Bristol-Myers Squibb and Baxter Healthcare. CS has received research funding from GlaxoSmithKline and AstraZeneca and is a founder of Exvastat. CP is a member of Advisory Boards of Immune Regulation Ltd, Eurodrug, Sativa, holds NED positions with Immune Regulation Ltd, PrEP Biopharm, EpiEndo and Babraham Biotechnology Ltd, a trustee of Babraham Institute and Fraunhofer Institute of Experimental Medicine and Toxicology and holds equity in Immune Regulation Ltd, and Verona Pharma. DS has received research grants/personal fees/travel from AstraZeneca, Boehringer Ingelheim, Chiesi, Cipla, Genentech, GlaxoSmithKline, Glenmark, Menarini, Mundipharma, Novartis, Peptinnovate, Pfizer, Pulmatrix, Teva, Therevance and Verona. GG has received research grants from Jannsenn-Cilag, AstraZeneca, Novartis Pharma, Astex, Roche, Heartflow, Celldex, Bristol-Myers Squibb and BioNTech. JC has received research grants/ personal fees from Astrazeneca, Boehringer Ingelheim, Glaxosmithkline, Insmed and Gilead Sciences. LM has received research grants/personal fees/ travel from Boehringer Ingelheim, Chiesi, GlaxoSmithKline, Merck, AstraZeneca, Bionorica, Vernalis, Afferent, Merck, Bellus, Bayer, Celerio \& Sanofi. L-PH has received research grants from Boehringer Ingleheim and Celgene. $\mathrm{KL}$ is Medical Director of Respiratory Innovation Wales and a Director of Bond Digital Health and lomics Ltd and has received sponsorship to attend and speak at international meetings from AstraZeneca, Boehringer Ingelheim, GlaxoSmithKline and Pfizer. TF has received sponsorship to attend and speak at international meetings, honoraria for lecturing or attending advisory boards, from Gilead, Pfizer and Menarini and is an advisor to the NICE/NHSE AMR novel antimicrobial value assessment/reimbursement project stakeholder advisory group. TW has received research grants/personal frees/travel from AstraZeneca, Boehringer Ingelheim, Chiesi, GlaxoSmithKline, Synairgen \& mymhealth.

\section{Author details}

${ }^{1}$ University of Southampton, Southampton, Hampshire, UK. ${ }^{2} \mathrm{NIHR}$ Southampton Biomedical Research Centre, Southampton, UK. ${ }^{3} \mathrm{IQVIA}$, Reading, UK. ${ }^{4}$ King's College London, London, UK. ${ }^{5}$ Public Health England, London, UK. ${ }^{6} \mathrm{NIHR}$ Oxford Biomedical Research Centre, University of Oxford, Oxford, UK. ${ }^{7}$ Population Health Sciences Institute, Faculty of Medical Sciences, Newcastle University, Newcastle upon Tyne, UK. ${ }^{8}$ Manchester NIHR Biomedical Research Centre, University of Manchester, Manchester, UK. ${ }^{9}$ Swansea University, Swansea, UK. ${ }^{10}$ University Hospital Southampton NHS Foundation Trust, Southampton, UK. ${ }^{11}$ University of Dundee, Dundee, UK. ${ }^{12}$ Imperial College London, London, UK. ${ }^{13}$ Queen's University Belfast, Belfast, UK. ${ }^{14}$ Evelina London Children's Hospital Guy's and St Thomas' Hospital NHS Foundation Trust, London, UK. ${ }^{15} \mathrm{IQVIA}$, Dublin, Ireland. ${ }^{16} \mathrm{IQVIA}$, Edinburgh, UK. ${ }^{17}$ University of Cambridge, Cambridge, UK. ${ }^{18}$ University of Manchester, Manchester, UK

Received: 1 July 2020 Accepted: 4 July 2020

Published online: 31 July 2020

\section{Publisher's Note}

Springer Nature remains neutral with regard to jurisdictional claims in published maps and institutional affiliations.

Ready to submit your research? Choose BMC and benefit from:

- fast, convenient online submission

- thorough peer review by experienced researchers in your field

- rapid publication on acceptance

- support for research data, including large and complex data types

- gold Open Access which fosters wider collaboration and increased citations

- maximum visibility for your research: over $100 \mathrm{M}$ website views per year

At BMC, research is always in progress.

Learn more biomedcentral.com/submissions 